\title{
Intact Long-Term Potentiation but Reduced Connectivity between Neocortical Layer 5 Pyramidal Neurons in a Mouse Model of Rett Syndrome
}

\author{
Vardhan S. Dani and Sacha B. Nelson \\ Biology Department, Brandeis University, Waltham, Massachusetts 02453
}

\begin{abstract}
Mutations in MECP2 cause Rett syndrome and some related forms of mental retardation and autism. Mecp2-null mice exhibit symptoms reminiscent of Rett syndrome including deficits in learning. Previous reports demonstrated impaired long-term potentiation (LTP) in slices of symptomatic Mecp2-null mice, and decreased excitatory neurotransmission, but the causal relationship between these phenomena is unclear. Reduced plasticity could lead to altered transmission, or reduced excitatory transmission could alter the ability to induce LTP. To help distinguish these possibilities, we compared LTP induction and baseline synaptic transmission at synapses between layer 5 cortical pyramidal neurons in slices of wild-type and Mecp2-null mice. Paired recordings reveal that LTP induction mechanisms are intact in Mecp2-null connections, even after the onset of symptoms. However, fewer connections were found in Mecp2-null mice and individual connections were weaker. These data suggest that loss of $\mathrm{MeCP} 2$ function reduces excitatory synaptic connectivity and that this precedes deficits in plasticity.
\end{abstract}

\section{Introduction}

Rett syndrome (RTT) (Mendelian Inheritance in Man number 312750 ) is a neurodevelopmental disorder usually caused by mutations in the X-linked gene, methyl CpG binding protein-2 (MECP2) (Amir et al., 1999; Chahrour and Zoghbi, 2007). Affected girls appear to develop normally for the first 6-18 months but then begin displaying symptoms that include profound impairment of learning and memory, motor abnormalities, and autistic behavior (Hagberg, 1997). Affected boys can exhibit a similar range of symptoms, often with a rapid and severe course (Villard, 2007). Mice lacking functional Mecp2 (Mecp2-nulls) show neurological symptoms reminiscent of RTT (Chen et al., 2001; Guy et al., 2001). Hemizygous null males develop symptoms rapidly after 4 weeks, including severe motor and respiratory abnormalities, learning deficits, and lethality (Stearns et al., 2007). Postnatal MeCP2 overexpression in forebrain neurons or reactivation of a silenced allele rescues many symptoms of Mecp2null mice (Giacometti et al., 2007; Guy et al., 2007).

Previously, we observed reduced circuit activity in neocortical slices of Mecp2-null mice attributable to a shift in the balance between synaptic excitation and inhibition onto layer 5 pyramidal neurons (Dani et al., 2005). However, these changes could not be completely accounted for by the subtle changes in frequency and amplitude of miniature EPSCs (mEPSCs). In dissociated hippocampal neuron cultures and autaptic cultures from neona-

\footnotetext{
Received March 1, 2009; revised July 20, 2009; accepted July 22, 2009.

This work was supported by grants from the National Institute of Neurological Disorders and Stroke, the McKnight Foundation, and the International Rett Syndrome Research Foundation. We thank Zhe Meng and Hao Fan for help with genotyping, as well as Gina Turrigiano, Arianna Maffei, Chris Hempel, Ken Sugino, and Praveen Taneja for helpful comments.

Correspondence should be addressed to Sacha B. Nelson, Biology Department MS-008, Brandeis University, 415 South Street, Waltham, MA 02454. E-mail: nelson@brandeis.edu.

V. S. Dani's present address: Department of Biological Sciences, Stanford University, Stanford, CA 94305. DOI:10.1523/JNEUROSCI.1019-09.2009

Copyright $\odot 2009$ Society for Neuroscience ～0270-6474/09/2911263-08\$15.00/0
}

tal Mecp2-null mice, larger reductions in mEPSC frequency and evoked EPSC amplitude have been reported (Nelson et al., 2006; Chao et al., 2007), but whether these changes involve changes in release probability or in the number of release sites seems to depend on preparation. Synaptic marker staining intensity is reduced in hippocampal slices of 2-week-old Mecp2-null mice, but not in symptomatic, 5-week-old Mecp2-null mice (Chao et al., 2007), leaving open the question of precisely which functional changes in synaptic properties occur in vivo.

Deficits in long-term potentiation (LTP) at neocortical and hippocampal synapses have been reported in Mecp2-mutant mice (Asaka et al., 2006; Moretti et al., 2006). However, LTP induction using extracellular stimulation is notoriously dependent on the strength of excitatory stimulation and on the balance between excitation and inhibition (Artola and Singer, 1987; Bear et al., 1992; Bliss and Collingridge, 1993; Sjöström et al., 2001). Moreover, the highly recurrent nature of neocortical circuits complicates interpretation of extracellular field potentials and renders analysis of synapse-specific potentiation less straightforward than in some circuits. Determining whether an initial plasticity deficit alters the circuit, or whether a change in circuitry alters plasticity induction, may hold important mechanistic implications for how loss of Mecp2 disrupts forebrain function.

To directly address the mechanisms underlying altered synaptic transmission and plasticity in local neocortical circuits, we studied unitary synaptic connections between layer 5 (L5) thick-tufted pyramidal neurons using quadruple whole-cell recordings in cortical slices from 2- to 4-week-old Mecp2-null and wild-type mice. We observed reduced connectivity and weaker synapses in slices of 4-week-old Mecp2-null mice, whereas comparable LTP could be induced in both conditions. Hence an early effect of Mecp 2 mutation is to reduce cortical excitatory synapses through a mechanism distinct from blocking LTP. 


\section{Materials and Methods}

Animals. Male wild-type (WT) controls and Mecp2-null mice were obtained by crossing females heterozygous for the Mecp $2^{1 \text { lox }}$ allele (Chen et al., 2001) and male C57BL/6 (The Jackson Laboratory). Mothers of all animals used in this study were progeny of backcrossings with C57BL/6 mice over more than six generations. All procedures for animal handling and killing were approved by the Animal Care and Use Committee of Brandeis University.

Solutions. The artificial CSF (ACSF) used for brain slicing contained the following (in $\mathrm{mm}$ ): $125 \mathrm{NaCl}, 2.5 \mathrm{KCl}, 3 \mathrm{MgCl}_{2} \cdot 6 \mathrm{H}_{2} \mathrm{O}, 1.25$ $\mathrm{NaHPO}_{4}, 25 \mathrm{NaHCO}_{3}, 1 \mathrm{CaCl}_{2} \cdot 2 \mathrm{H}_{2} \mathrm{O}, 14 \mathrm{dex}-$ trose. For incubation of slices and for recordings, ACSF was modified as follows (in $\mathrm{mM}$ ): 1 $\mathrm{MgCl}_{2} \cdot 6 \mathrm{H}_{2} \mathrm{O}, 2 \mathrm{CaCl}_{2} \cdot 2 \mathrm{H}_{2} \mathrm{O}$. ACSF was continuously bubbled with a 95:5 mixture of $\mathrm{O}_{2}: \mathrm{CO}_{2}$. For whole-cell patch-clamp recordings, pipette internal solution contained the following (in $\mathrm{mm}$ ): $20 \mathrm{KCl}, 100 \mathrm{~K}$-gluconate, 10 HEPES, $0.2 \%(\mathrm{w} / \mathrm{v})$ biocytin, $4 \mathrm{Mg}$-ATP, 0.3 $\mathrm{Na}-\mathrm{GTP}$, and $10 \mathrm{Na}_{2}$-phosphocreatinine.

Brain slices. Anesthetized animals were decapitated, and the brain was removed under cold ACSF. Coronal brain slices ( $300 \mu \mathrm{m}$ thick) were obtained from 2- to 4-week-old animals on a Leica VT1000S (Leica Microsystems). Slices were incubated initially at $35^{\circ} \mathrm{C}$ for $15 \mathrm{~min}$ and then kept at room temperature.

Electrophysiology. Slices were continuously perfused with oxygenated ACSF maintained at $31-33^{\circ} \mathrm{C}$ with a TH324B in-line solution heater and controller (Warner Instruments). Somatic whole-cell patch-clamp recordings were obtained by patching up to four visually identified thick-tufted L5 pyramidal neurons in the primary somatosensory cortex. Patch pipettes were pulled from borosilicate capillaries (World Precision Instruments) on a Sutter P-97 Flaming/Brown puller (Sutter Instruments). The somas of cells targeted for patching were typically within $50 \mu \mathrm{m}$ of each other. After breakthrough of cell membrane, resting membrane properties and access resistance were checked. Identity of cell type was confirmed by testing for a nonadapting action potential (AP) firing type (Hattox and Nelson, 2007) and post hoc staining for biocytin. Data, acquired with Multiclamp 700A and 700B amplifiers (Molecular Devices), were low-pass filtered at $3 \mathrm{kHz}$ and digitized at $10 \mathrm{kHz}$. Recordings were acquired and analyzed using Igor Pro software (Wavemetrics) and in-house programs.

Trains of 5-ms-long depolarizing current pulses $(0.7-1.2 \mathrm{nA})$ at $20 \mathrm{~Hz}$ elicited timed presynaptic action potentials, and were repeated at 0.056 $\mathrm{Hz}$, throughout the recording (except during LTP induction). Monosynaptic connections were identified when the off-line average of at least 10 repetitions in any candidate postsynaptic neuron showed EPSPs time locked with the train of presynaptic action potentials, and if the EPSP amplitude in the averaged waveform was at least $100 \mu \mathrm{V}$. Thus, most monosynaptic connections could be easily identified (supplemental Fig. 1, available at www. jneurosci.org as supplemental material), but those with extremely high failure rates or low initial strength were most likely excluded.

After acquiring at least 30 baseline synaptic responses, LTP was induced with trains of five precisely timed action potentials at $40 \mathrm{~Hz}$, repeated 20 times at $0.1 \mathrm{~Hz}$, such that each postsynaptic AP followed the presynaptic AP by $8-10 \mathrm{~ms}$. This spike timing relationship between presynaptic and postsynaptic firing is known to induce LTP at neocortical excitatory synapses (Markram et al., 1997a; Feldman, 2000; Sjöström et al., 2001). Recordings were included for LTP analysis only if presynaptic and postsynaptic cells remained stable for at least $35 \mathrm{~min}$ from the start of the baseline period, typically up to $50 \mathrm{~min}$ (70 $\mathrm{min}$ in some cases). Neurons were discarded from analysis if a $>25 \%$ change in resting membrane potential $\left(V_{\mathrm{m}}\right)$, series or input resistance $\left(R_{\mathrm{s}}\right.$ or $\left.R_{\mathrm{in}}\right)$ was observed.

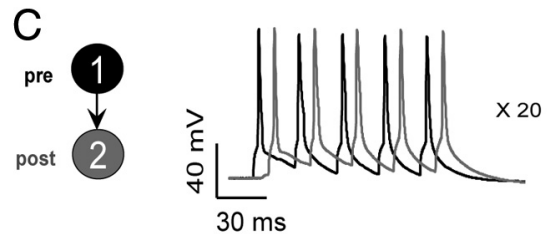

$\mathrm{D}$
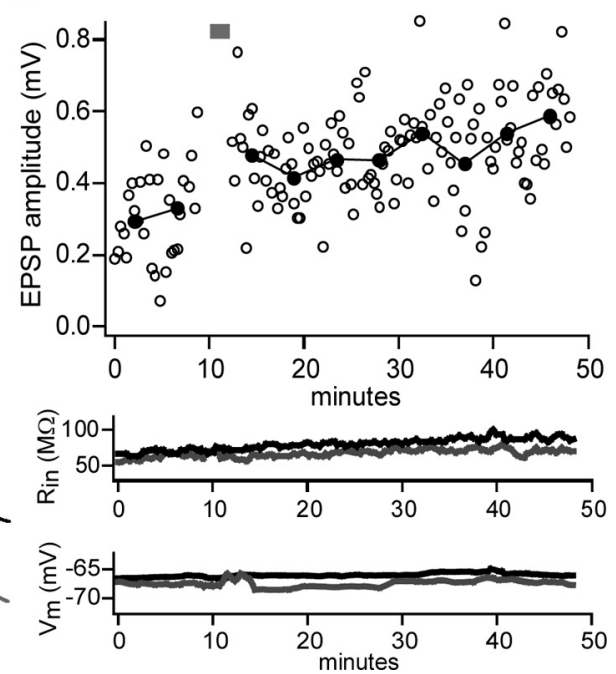

Figure 1. Whole-cell recordings from monosynaptically connected layer 5 thick-tufted neurons. $\boldsymbol{A}$, Pseudocolor (grayscale) confocal z-stack projection of four simultaneously patched cells filled with Alexa 594. B, Example monosynaptic responses (gray ace; average of 30 trials) time locked to presynaptic APs (black trace). C, Induction of spike-timing-dependent LTP by repeated of 5 ) throughout the recording. The gray bar indicates time during which LTP was induced. Input resistance $\left(R_{\text {in }}\right)$ (center) and (bottom) changed little through the duration of recording.

A "pairing" protocol was also used to induce LTP (Malinow, 1991; Chen et al., 1999). An extracellular stimulating electrode consisting of an ACSF-filled pipette $(0.7-2 \mathrm{M} \Omega$ ) was placed $10-50 \mu \mathrm{m}$ from the patched L5 pyramidal neuron soma. Fibers passing through layer 5 were stimulated with bipolar current pulses $(10-70 \mu \mathrm{A} ; 0.1-0.5 \mathrm{~s})$ using a linear stimulus isolator (A395; World Precision Instruments). Evoked EPSCs were measured while holding the postsynaptic neuron at $-70 \mathrm{mV}$ in voltage clamp. Only those responses that did not have a component that reversed at $-45 /-40 \mathrm{mV}$ were recorded further to test for LTP induction. During each induction trial, presynaptic stimulation at $2 \mathrm{~Hz}$ was paired with postsynaptic depolarization to $0 \mathrm{mV}$ for $5 \mathrm{~s}$, and then repeated 20 times at $0.1 \mathrm{~Hz}$. GABAergic inhibition was not blocked.

Synaptic strength (mean EPSP amplitude) and failure rate were calculated for the first EPSP amplitude in the train during the preinduction/baseline period. EPSP waveform properties were quantified from the average waveform for the first EPSP in the train. Rise time was defined as the time from 20 to $80 \%$ of peak amplitude, latency was defined as the time between the peak of the presynaptic AP and the onset of the EPSP (defined at $5 \%$ of peak), and decay time constant was obtained by fitting a single exponential to the decaying phase of the EPSP. Short-term plasticity was assessed as the pairedpulse ratio (PPR) computed as follows: $\mathrm{PPR}=\mathrm{EPSP} i / \mathrm{EPSP} 1$.

The connection probability $(\mathrm{CP})$ was expressed as the ratio of the number of identified synaptic connections to the number of synaptic connections tested. Difference in CP was tested by a $\chi^{2}$ test for a $2 \times 2$ contingency table, in which rows consisted of the numbers of connected and unconnected pairs and columns consisted of the genotype (WT and Mecp2-null).

All bar plots represent mean values \pm SEM.

\section{Results}

Quadruple whole-cell recordings from L5 pyramidal neurons (Fig. $1 A$ ) were performed to study monosynaptic connections in slices from both WT and Mecp2-null animals. Evoked EPSPs were identified as depolarizing responses time locked to presyn- 
A

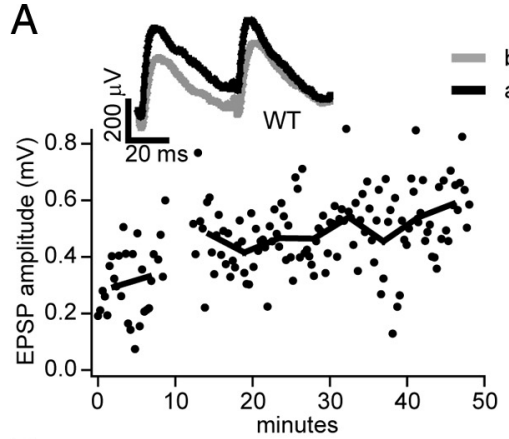

B

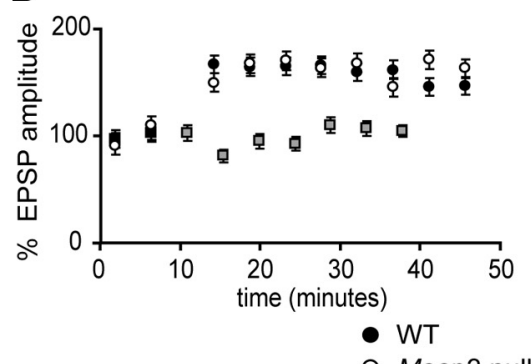

○ Mecp2-null $\square \mathrm{WT}$, no induction

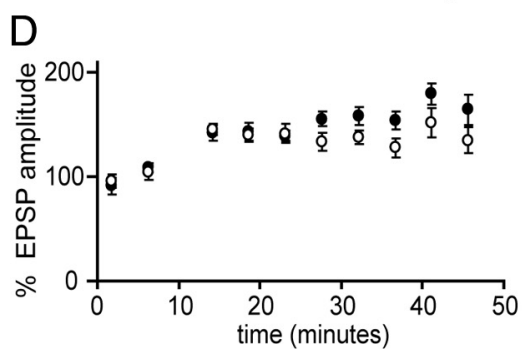

$\mathrm{E}$

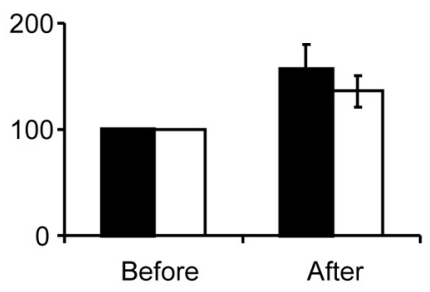

Figure 2. Robust LTP of monosynaptic connections between L5 pyramids in WT and Mecp2-null slices. A, Sample LTP experiments for WT (left; filled circles) and Mecp2-null (right; open circles) connections. Individual trials in each 4.5 min period were averaged (solid line); error bars were omitted for clarity. The insets show average ( $n=30$ trials) of first two EPSPs in the train during baseline (gray) and postinduction (black; 25 th to 35 th minutes) periods. $B$, Magnitude of LTP was similar in pairs from 2to 3-week-old WT ( $N=14$ ) and Mecp2-null ( $N=11)$ mice. The plot shows preinduction baseline normalized EPSP amplitude for the first response in the train. Each point represents mean \pm SEM of 15 consecutive trails. Recordings with no induction protocol are also plotted (gray squares; $N=3$ ). C, Bar plot showing average normalized baseline responses ("Before"; 30 trials per pair) from $B$ and average of same number of responses centered around the 30 th minute of each recording ("After"; 30 trials per pair). $D, E$, Summary of LTP experiments done in slices obtained from 4-week-old mice (WT, $N=8$ connections; Mecp2-null, $N=7$ connections). No significant difference in LTP magnitude between WT and Mecp2-null pairs was present at either developmental stage. Error bars indicate SEM.

aptic spikes (Fig. 1B). A set of baseline EPSPs were used to study properties of evoked synaptic transmission in WT and Mecp2null conditions. LTP was induced using a spike-timing-dependent plasticity (STDP) protocol (see Materials and Methods) (Fig. 1C). A connection was considered potentiated if the mean postinduction EPSP amplitude (from 30 consecutive trials averaged around the 30th minute, from start of recording) was $>120 \%$ of the mean baseline EPSP amplitude, and if this increase was statistically significant by a $t$ test. Input resistance and resting membrane potential were monitored throughout the recording (Fig. $1 D$, center and bottom graphs, respectively).

\section{LTP induction is unchanged in connections between L5} pyramidal neurons

Previously, we observed an early reduction in circuit excitability of 2- to 3-week-old Mecp2-null mice (Dani et al., 2005). Therefore, we first studied monosynaptic pyramid-pyramid connections in slices from presymptomatic [postnatal day 16 (P16) to P21] Mecp2-null mice and WT controls. Synaptic connections in both WT and Mecp2-null mice could be successfully potentiated using the STDP protocol (Fig. 2). Averages of normalized EPSPs from all connections, for which LTP was attempted, revealed no significant differences in plasticity between WT and Mecp2-null mice (Fig. 2B,C). To determine whether or not LTP induction was impaired later in development, during the onset of primary symptoms in male Mecp2-null mice, we performed LTP experiments in slices obtained from 4-weekold (P26-P29) WT and Mecp2-null mice (supplemental Fig. 2, sample LTP experiments, available at www.jneurosci.org as supplemental material). A few recordings in older animals were attempted, but analysis of LTP proved impractical because of the increased difficulty of finding and maintaining stable paired recordings in slices from older animals. There were also no significant differences in the magnitude of LTP in slices obtained from 4-week-old WT and Mecp2-null animals (Fig. 2D,E) (WT, $157 \pm 23 \%$; Mecp2-null, $136 \pm 12 \%$; $p=0.43$, Student's $t$ test). Analysis of changes in the inverse square of the coefficient of variation $\left(\mathrm{CV}^{-2}\right)$ relative to changes in mean EPSP amplitude can provide information about relative changes in quantal content (usually presynaptic) or quantal amplitude (often postsynaptic) (Malinow and Tsien, 1990; Faber and Korn, 1991; Larkman et al., 1992; Sjöström et al., 2003, 2007). We have shown previously that two apparently presynaptic and postsynaptic forms of LTP coexist at L5 synapses in rat visual cortex and that this leads to changes in short-term plasticity and CV that vary between synaptic connections but are concordant at any one connection (Sjöström et al., 2007). Such an analysis for WT and Mecp2-null connections did not reveal major differences in the locus of LTP expression between WT and Mecp2-null connections at any age (supplemental Fig. 3, available at www.jneurosci.org as supplemental material). To ensure that the inability to see differences in LTP induction was not specific to the STDP induction protocol, we also used a pairing protocol in which extracellular stimulation was used to activate excitatory inputs during strong depolarization of the postsynaptic neuron (Fig. $3 A, B$ ). This protocol is known to produce robust strengthening of excitatory synapses (Malinow, 1991; Chen et al., 1999). Consistent with previous observations, pairing induced LTP had a much greater amplitude than that observed following the STDP protocol (Malinow, 1991; Markram et al., 1997a; Chen et al., 1999; Sjöström et al., 2001); however, no significant difference in LTP existed between synapses from WT and Mecp2-null slices using this protocol either (Fig. 3C). Thus, even during the early symptomatic phase of the mouse disorder, the signaling mechanisms required for longterm strengthening of synapses seem to be intact in Mecp2-nulls, at least for synapses on to L5 neurons. 

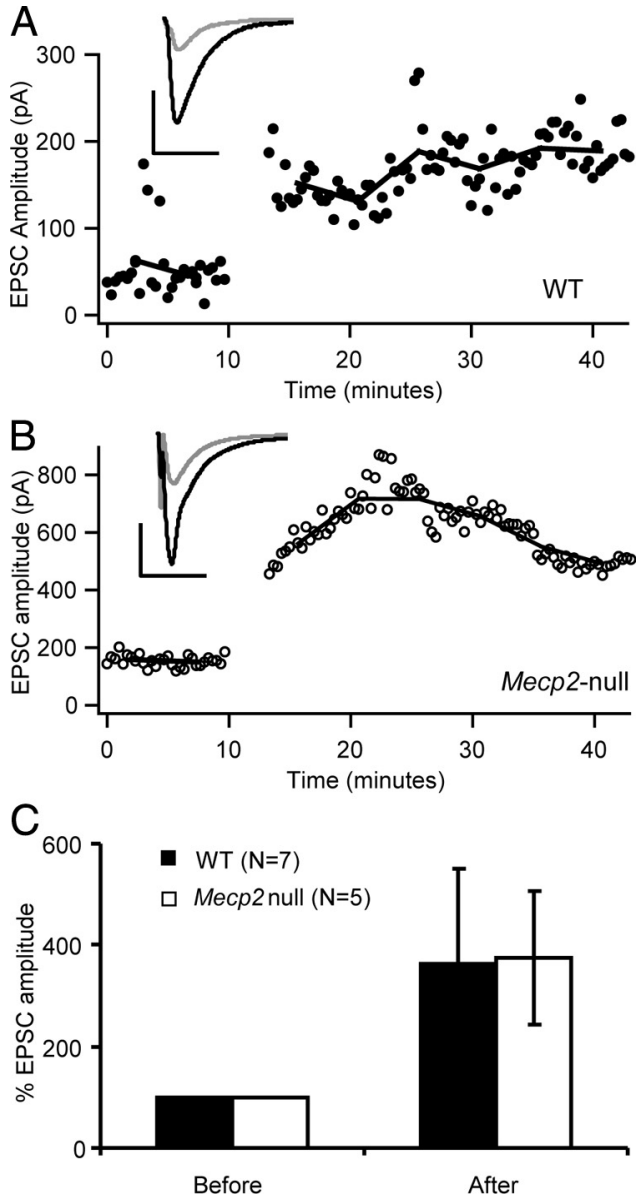

Figure 3. LTP induction using a pairing protocol. Evoked EPSCs were measured in L5 pyramidal neurons voltage clamped at $-70 \mathrm{mV}$. $A$, Example of an LTP experiment in a WT slice. The inset shows averaged EPSC traces before (gray) and after (black) induction. Calibration: $100 \mathrm{pA}$ $20 \mathrm{~ms}$. $\boldsymbol{B}$, Example of a Mecp2-null neuron that underwent potentiation. The inset shows averaged EPSC traces before (gray) and after (black) induction. Calibration: 200 pA, 20 ms. C, Summary of LTP experiments using pairing protocol. Bar plot of mean normalized EPSC amplitude before (0 to 10th minute) and after (25th to 30th minute) pairing. Both WT and Mecp2-null synapses undergo strong potentiation when postsynaptic depolarization is provided. Error bars indicate SEM.

\section{Baseline synaptic transmission is normal in presymptomatic} Mecp2-null mice

Differences in the properties of evoked synaptic transmission between neurons from WT and Mecp2-null slices were analyzed from baseline synaptic responses acquired before LTP induction. There were no differences in the mean amplitude of the first EPSP (Fig. $4 B$ ) (WT, $0.67 \pm 0.1 \mathrm{mV} ; n=36$; Mecp2-null, $0.58 \pm 0.08$ $\mathrm{mV}, n=24 ; p=0.46$, two-tailed, unpaired Student's $t$ test) or the rate of failures (Fig. 4C) (percentage of failures, WT, $14.9 \pm 3.0 \%$; Mecp2-null, $18.8 \pm 2.8 \%$ ) between the two conditions. The average latency, rise time, and decay time constants for the first EPSP in the train were comparable between the two genotypes (supplemental Table T1, available at www.jneurosci.org as supplemental material). There were also no significant differences in the shortterm plasticity of these synapses as quantified by the PPR for a train of three pulses (Fig. 4D). A train of five APs was delivered to a subset of these connections, but, again, no significant differences were observed (supplemental Fig. 4, available at www. jneurosci.org as supplemental material). The $\mathrm{CV}^{-2}$, which is proportional to quantal content assuming a binomial model of synaptic transmission, was not significantly different for any of
A
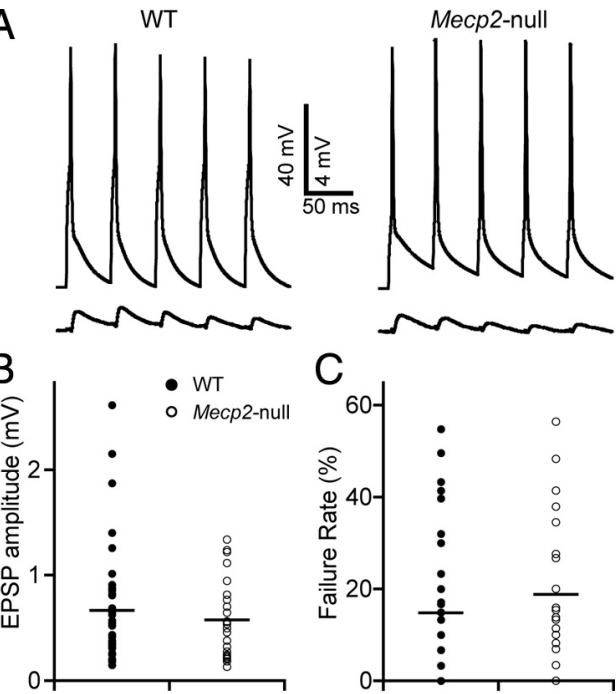

D

E
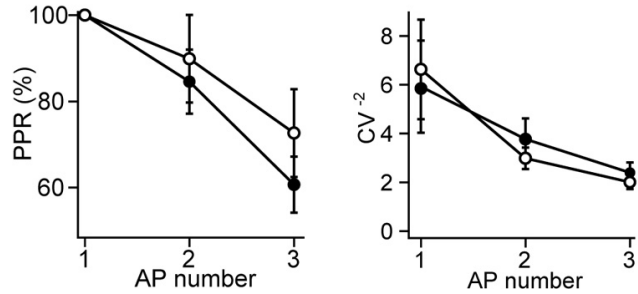

Figure 4. Properties of baseline synaptic transmission in $L 5$ pyramid-pyramid connections in 2- to 3-week-old WT and Mecp2-null mice. $\boldsymbol{A}$, Sample average postsynaptic responses of a synaptic connection in WT (left) and Mecp2-null (right) slices, and corresponding presynaptic spikes. $\boldsymbol{B}$, EPSP amplitudes for the first EPSP in the train for each connection in WT (filled circles; $N=36$ pairs) and Mecp2-null (empty circles; $N=24$ pairs) mice. Horizontal bars show mean EPSP amplitude. C, No significant difference between genotypes was observed in the mean failure rates for the first EPSP in the train (circles, individual pairs; horizontal bars, mean failure rate for each genotype). $\boldsymbol{D}$, Short-term plasticity of EPSPs in a train of three presynaptic action potentials delivered at $20 \mathrm{~Hz}$. Mean percentage change ( \pm SEM) with respect to first EPSP in the train (paired-pulse ratio) is plotted for each AP number. Both WT (filled circles) and Mecp2-null (open circles) synapses appear to depress to the same extent. $\boldsymbol{E}$, No significant differences in the mean $\mathrm{CV}^{-2}$ (quantal content) can be observed between WT and Mecp2-null conditions during a train of APs. Each point represents mean $\mathrm{CV}^{-2} \pm$ SEM.

the three stimuli in the train (Fig. $4 E, \mathrm{CV}^{-2}$ ). Thus, at L5 pyramid-pyramid connections, there are no major differences in the properties of synaptic transmission in slices obtained from presymptomatic, 16- to 21-d-old animals.

Weaker excitatory synapses in 4-week-old Mecp2-null mice Since more dramatic effects in spontaneous firing rates in L5 pyramids accompanied by reduced excitatory synaptic drive were previously observed in slices from 4- to 5-week-old Mecp2-null mice, we analyzed properties of baseline synaptic transmission in 4-week-old (P26-P29) animals (Fig. 5A, sample traces of WT and Mecp2-null monosynaptic connections). The mean EPSP amplitude of L5 pyramid-pyramid synapses in Mecp2-nulls was reduced by nearly $45 \%$ compared with WT (Fig. 5B) (WT, $0.66 \pm 0.13 \mathrm{mV}, n=19 ;$ Mecp2 null, $0.36 \pm 0.03 \mathrm{mV}, n=18$; $p<0.05$, two-tailed unpaired Student's $t$ test). The apparent increase in the mean failure rate of Mecp2-null synapses was not statistically significant (Fig. 5C) (percentage of failures, WT, $19.9 \pm 3.6 \%, n=19 ;$ Mecp2-null, $27.2 \pm 4.2 \%, n=18 ; p=0.19$, two-tailed Student's $t$ test). Mean EPSP latency, rise time, and decay kinetics were also not significantly different between WT 


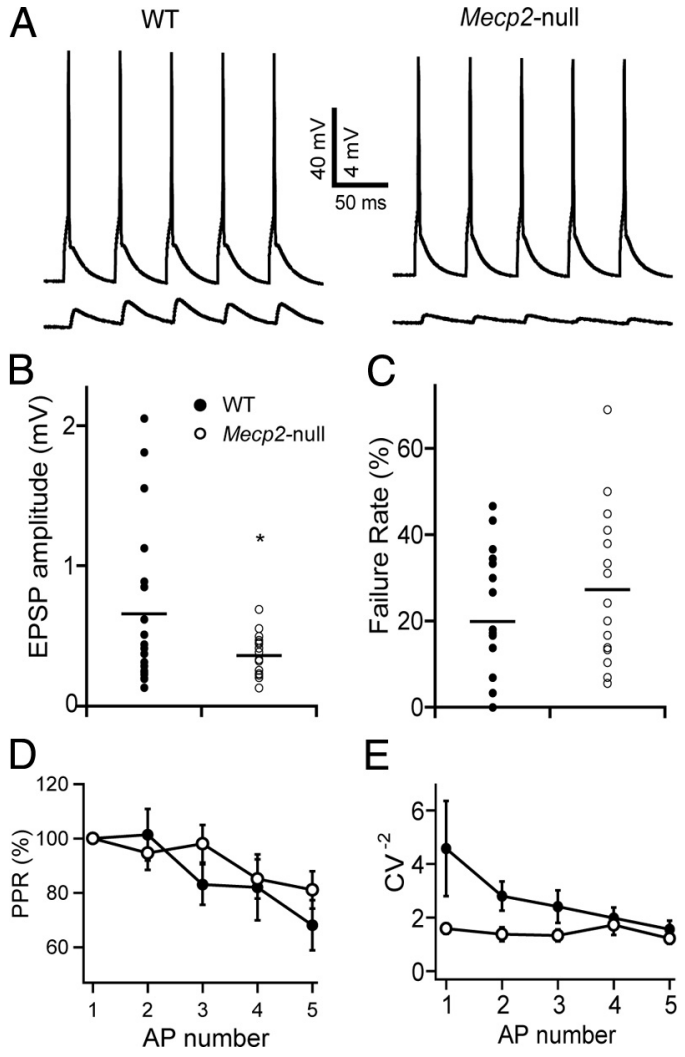

Figure 5. Properties of baseline synaptic transmission in $\mathrm{L} 5$ pyramid-pyramid connections in 4-week-old WT and Mecp2-null mice. A, Sample average postsynaptic responses of a monosynaptic connection in WT (left) and Mecp2-null (right) slices and corresponding presynaptic spikes (black). $\boldsymbol{B}$, EPSP amplitudes for the first EPSP in the train for each connection in WT (filled circles) and Mecp2-null (empty circles) mice. Mean EPSP amplitude (horizontal bar) is $\sim 45 \%$ smaller in the Mecp2-null $(N=18)$ condition compared with WT $(N=19)$. The asterisk $\left({ }^{*}\right)$ indicates statistical significance $(p<0.05)$ by unpaired Student's $t$ test. $C$, The apparent increase in mean failure rate (horizontal bars) was not statistically significant ( $p=0.19$; unpaired Student's $t$ test). Failure rates for individual WT (filled circles) and Mecp2-null (open circles) synaptic connections are also shown. $\boldsymbol{D}$, Short-term plasticity of EPSPs in a train of five pulses delivered at $20 \mathrm{~Hz}$, plotted as PPR (percentage \pm SEM) for each AP in the train. $\boldsymbol{E}$, Mean $\mathrm{CV}^{-2}\left( \pm\right.$ SEM) plotted for each EPSP in the train. $\mathrm{CV}^{-2}$ is higher for WT synapses and decreases with successive stimulation. At Mecp2-null synapses, $\mathrm{CV}^{-2}$ for the first two EPSPs in the train is significantly lower compared with WT and does not change significantly with successive stimuli ( $p<0.05$, two-factor ANOVA between WT and Mecp2-null curves).

and Mecp2-null synapses (supplemental Table T1, available at www.jneurosci.org as supplemental material). As in younger animals, no significant changes were observed in short-term plasticity with repeated stimulation at $20 \mathrm{~Hz}$ (Fig. 5D) (WT, $n=19$; Mecp2-null, $N=18 ; p=0.52$, one-way ANOVA). However, WT synapses had significantly higher $\mathrm{CV}^{-2}$ during the first two EPSPs in the train compared with Mecp2-null synapses. In addition, consistent with short-term depression of quantal content, $\mathrm{CV}^{-2}$ decreased on average during a train of EPSPs for WT synapses, but for Mecp2-null synapses it did not change significantly (Fig. $5 E)\left[\mathrm{CV}^{-2}\right.$ for WT $(N=18)$ and Mecp2-null $(N=18)$ synapses; $p<0.05$, two-factor ANOVA]. Thus, the reduced excitatory synaptic strength in Mecp2-null mice at an early symptomatic phase could be partially explained by reduced quantal content at these synapses.

\section{Reduced connectivity in Mecp2-null mice}

The CP at both developmental time points was computed for all potential connections tested in WT and Mecp2-null slices. Simi-
Table 1. Reduced connection probability in Mecp2-null mice

\begin{tabular}{llll}
\hline & Pairs tested & No. connections & Connection probability (\%) \\
\hline P16-P21 & & & \\
WT & 418 & 40 & 9.6 \\
Mecp2-null & 322 & 25 & 7.8 \\
P26-P29 & & & \\
WT & 208 & 21 & 10.1 \\
Mecp2-null & 418 & 22 & $5.3^{*}$ \\
\hline
\end{tabular}

Connection probability was calculated as the ratio of the number of connections found to the number of connection tested. Although a small reduction in connectivity was observed in 2- to 3-week-old mice, this difference was not statistically significant ( $p>0.25, \chi^{2}$ test). A significant reduction in connection probability $(\sim 50 \%)$ was observed in 4-week-old Mecp2-null mice compared with WT ( ${ }^{*} p<0.05, \chi^{2}$ test).

lar to previous estimates for synapses between thick-tufted, L5 pyramidal neurons in rat and mouse somatosensory and visual cortices (Markram et al., 1997b; Song et al., 2005; Krieger et al., 2007 ), the rate of finding paired connections was $\sim 10 \%$ in 2 - to 4-week-old WT mice. Although CP was lower in 2- to 3-week-old Mecp2-null mice compared with WT (Table 1), this difference was not significant ( $p>0.25$; two-sided $\chi^{2}$ contingency test). CP in slices from 4-week-old Mecp2-null mice was found to be nearly one-half of that in WT slices (Table 1), and this difference was statistically significant ( $p<0.05, \chi^{2}$ test). This suggests that one primary effect of Mecp 2 mutation is to reduce excitatory connectivity within local cortical microcircuits.

\section{Discussion}

To determine which properties of baseline excitatory synaptic transmission and synaptic plasticity are altered because of the loss of MeCP2 function, we recorded from pairs of synaptically connected pyramidal neurons, before and during the onset of symptoms in Mecp2-null mice. Our results reveal a progressive reduction in excitatory connectivity in the neocortex, reminiscent of that described at hippocampal autapses in vitro (Chao et al., 2007). However, our results also suggest that a reduced ability to induce LTP at cortical synapses is likely to be a late consequence of loss of MeCP2 and may be secondary to changes in circuit structure.

\section{LTP induction is normal in developing Mecp2-null mice}

Despite the fact that L5 neocortical pyramidal neurons in Mecp2nulls show reduced spontaneous activity (Dani et al., 2005) and reduced number and strength of local recurrent excitatory synapses (Fig. 5, Table 1), LTP induction and expression appear to be intact at these synapses when postsynaptic depolarization is provided directly. This is true at 2-3 weeks when subtle changes in network excitability can first be detected (Dani et al., 2005) and remains true at the onset of symptoms at $\sim 4$ weeks (Fig. 2 ), when more marked changes in synaptic strength and number are already apparent. This shows that the signaling pathways mediating long-term strengthening of excitatory synapses are unlikely to be initial targets of MeCP2 and that loss of LTP is unlikely to explain the reduced cortical connectivity. Previous studies using extracellular stimulation and recordings have reported deficits in the induction of LTP at hippocampal synapses of symptomatic Mecp2-null mice (Asaka et al., 2006) and Mecp $2^{308}$ mice (Moretti et al., 2006). Reduced LTP of cortical field EPSPs in layer $2 / 3$ was also observed in symptomatic 35- to 40-week-old Mecp $2^{308}$ mice (Moretti et al., 2006). Although it remains conceivable that there are early changes specific to L2/3 synapses, morphological abnormalities such as simplified dendritic arbors in L5 neurons parallel similar changes in many other excitatory neurons in the forebrain, including L2/3 neurons (Belichenko et al., 1994; Kishi and 
Macklis, 2004). Moreover, since defects in synaptic transmission and connectivity can be seen earlier in development (Nelson et al., 2006; Chao et al., 2007), reduced plasticity could be a late secondary effect of circuit miswiring.

Alternatively, reduced plasticity may reflect the procedure used to induce it. Previous studies of plasticity in Mecp2-mutant mice used protocols that rely on extracellular stimulation to achieve sufficient postsynaptic depolarization and calcium influx during LTP induction. In these protocols, synaptic plasticity is highly cooperative, requiring activation of multiple excitatory inputs to produce sufficient postsynaptic depolarization to allow the NMDA receptor-mediated $\mathrm{Ca}^{2+}$ influx required for potentiation (Bliss and Collingridge, 1993; Kirkwood and Bear, 1994; Debanne et al., 1996; Sjöström et al., 2001). Thus, progressively weaker synapses and sparser connectivity in late symptomatic stages of Mecp2-null mice may impair LTP induction when postsynaptic depolarization or firing is not directly controlled. Using two different methods for LTP induction-STDP and a pairing protocol-we observed comparable LTP induction at L5 excitatory synapses of Mecp2-null and WT mice when postsynaptic depolarization was provided. Furthermore, since local cortical circuits are made up of highly recurrent excitatory and inhibitory inputs, extracellular stimulation, even in the presence of low doses of GABAergic antagonists, often evokes a mixture of excitation and inhibition. GABAergic inhibition is known to potently block LTP at cortical excitatory synapses (Artola and Singer, 1987; Bear et al., 1992). Hence a shift in the balance between excitatory and inhibitory synaptic drives onto L5 pyramidal neurons to favor inhibition (Dani et al., 2005) could also contribute to impaired LTP induction after extracellular stimulation. During paired recording, this circuit-level "gate" on plasticity is presumably circumvented by directly providing postsynaptic firing during synaptic activation.

\section{Altered synaptic transmission and connectivity}

Previous studies have reported reduced spontaneous and evoked excitatory neurotransmission in cultured hippocampal pyramidal neurons from Mecp2-null mice but have differed on whether this was attributable to changes in release properties of individual synapses (Nelson et al., 2006) or changes in synapse number (Chao et al., 2007). Moreover, it was not known whether or not changes observed at hippocampal synapses also occur in the neocortex or whether observations made in neuronal cultures were recapitulated in vivo. The latter point is important because targets of $\mathrm{MeCP} 2$, like BDNF, may be regulated differently in culture and in vivo because of very different prevailing activity patterns (Chang et al., 2006). Our results are consistent with a reduction in the number of recurrent excitatory synapses in the neocortex of symptomatic mice similar to that reported for autaptic hippocampal synapses in culture (Chao et al., 2007). This would explain the nearly twofold reduction in connection probability observed (Table 1). It could also account for the nearly twofold decrease in mean EPSP amplitude observed at individual connections (Fig. 5B). However, we cannot rule out an additional change in the release properties of individual synapses, since each connection typically consists of multiple synaptic contacts (Markram et al., 1997b). The physiological evidence for reduced excitatory connectivity is consistent with anatomical evidence for reduced dendritic branching and reduced numbers of dendritic spines on cortical pyramidal neurons, especially in layers $2 / 3$ and 5 , reported in both Rett patients and Mecp2-null mice (Belichenko et al., 1994, 2009; Armstrong, 2002; Zoghbi, 2003; Kishi and Macklis,
2004; Fukuda et al., 2005). Despite the reduced ( 50\%) connectivity between L5 pyramids, mEPSCs recorded in the same cell type show only a mild reduction in frequency (Dani et al., 2005). However, mEPSC measurements are quite variable (CV for mEPSC frequency, WT, 45\%; Mecp2-null, 42\%) (from Dani et al., 2005) and represent population measures of all excitatory inputs from other layers and cell types. Thus, if L5 pyramidal neurons comprise only a small fraction of inputs onto neighboring L5 pyramids, even a 50\% reduction in local connectivity may not cause a sufficiently large difference in mEPSC frequency, if other excitatory inputs are unaltered. Additional studies will be required to test whether other excitatory inputs onto L5 pyramids (e.g., L2/3-to-L5 inputs) are altered in Mecp2-nulls.

The reduced probability of finding synaptic connections between pairs of L5 pyramidal neurons in Mecp2-null mice was more apparent in slices from symptomatic, 4-week-old Mecp2-null mice than in slices from younger mice. Connection probability of L5 pyramids in WT mice is constant over early postnatal development in our experiments (Table 1) and parallels similar observations in the rat somatosensory cortex (Frick et al., 2007). This suggests that cellular mechanisms involved in the maintenance of synaptic contacts during early postnatal development are perturbed in Mecp2-null mice. Recently, it has been shown that activity-dependent regulation of MeCP2 function is important for dendritic patterning and spine morphology (Zhou et al., 2006) and perturbation of MeCP2 levels can lead to altered activity-dependent structural synaptic plasticity. Moreover, results from cell type-specific gene expression profiling experiments indicate that a major class of dysregulated genes are involved in cell adhesion and synapse formation/maintenance (Sugino K, Hempel C, Arnson HA, Dani VS, and Nelson SB, unpublished observations).

Recurrent excitatory connectivity is critical for the amplification and processing of input signals to the neocortex (Douglas and Martin, 2004). Thus, loss of recurrent excitatory connections may cause aberrant intracortical processing of sensory-motor information and impaired output from layer 5 neurons to subcortical targets, many of which are crucial for motor control. Similar to the dendritic anomalies found in many regions of the forebrain of RTT patients and Mecp2-mutant mice (Armstrong, 2002; Zoghbi, 2003; Kishi and Macklis, 2004), loss of connectivity between L5 cortical pyramidal neurons reported here, is likely to be found in other forebrain regions. Therefore, such changes in circuit wiring could underlie several of the motor, cognitive, and learning deficits observed in Rett syndrome and related mental retardation disorders. However, studies of neocortical pyramidal neuron networks in the valproate model of autism in rats have revealed hyperconnectivity, rather than hypoconnectivity, although individual connections between pyramids were weaker, disynaptic inhibitory connections were stronger, and pyramidal neurons were less excitable (Markram et al., 2007; Rinaldi et al., 2008). Increased LTP was also observed at layer $2 / 3$ synapses in this model of autism (Rinaldi et al., 2007). Our results showing hypoconnectivity and intact LTP in pyramidal neuron networks suggest there may be important mechanistic differences underlying circuit miswiring in Rett syndrome, a monogenic autism spectrum disorder, and the valproate model for autism.

Fine-scale, cell type-specific changes in local circuit organization in the forebrain may be a common mechanism underlying several mental retardation disorders that lack overt signs of neurodegeneration or developmental malformations. In hippocampal slices from Ts65Dn mice (a model for Down syndrome), 
pharmacological blockade of inhibitory inputs has been shown to reverse a deficit in NMDA receptor-dependent LTP induction at hippocampal synapses (Kleschevnikov et al., 2004). Furthermore, study of monosynaptic connections between hippocampal CA3 neurons of Ts65Dn mice showed intact LTP induction mechanisms but alterations in excitatory and inhibitory synaptic connectivity (Hanson et al., 2007). Similarly, in a mouse model of fragile X syndrome, weaker interlaminar inputs (L4-L3) attributable to reduced connection probability and more diffuse axonal arborizations have been transiently observed, probably during a developmentally regulated critical period (Bureau et al., 2008). Thus, shifts in the excitation-inhibition balance caused by altered connectivity in forebrain circuits could be one underlying cause for learning and cognitive disabilities in several childhood mental retardation disorders including Rett syndrome. However, it remains to be determined whether or not these changes in connectivity are primary, cell-autonomous changes, or reflect secondary effects in response to altered function of other cell types. Furthermore, deciphering the underlying changes in gene expression caused by Mecp 2 mutation and primary targets of MeCP2 will be crucial to understanding the molecular mechanisms underlying altered neuronal connectivity in Rett syndrome.

\section{References}

Amir RE, Van den Veyver IB, Wan M, Tran CQ, Francke U, Zoghbi HY (1999) Rett syndrome is caused by mutations in X-linked MECP2, encoding methyl-CpG-binding protein 2. Nat Genet 23:185-188.

Armstrong DD (2002) Neuropathology of Rett syndrome. Ment Retard Dev Disabil Res Rev 8:72-76.

Artola A, Singer W (1987) Long-term potentiation and NMDA receptors in rat visual cortex. Nature 330:649-652.

Asaka Y, Jugloff DG, Zhang L, Eubanks JH, Fitzsimonds RM (2006) Hippocampal synaptic plasticity is impaired in the Mecp2-null mouse model of Rett syndrome. Neurobiol Dis 21:217-227.

Bear MF, Press WA, Connors BW (1992) Long-term potentiation in slices of kitten visual cortex and the effects of NMDA receptor blockade. J Neurophysiol 67:841-851.

Belichenko PV, Oldfors A, Hagberg B, Dahlström A (1994) Rett syndrome: 3-D confocal microscopy of cortical pyramidal dendrites and afferents. Neuroreport 5:1509-1513.

Belichenko PV, Wright EE, Belichenko NP, Masliah E, Li HH, Mobley WC, Francke U (2009) Widespread changes in dendritic and axonal morphology in Mecp2-mutant mouse models of Rett syndrome: evidence for disruption of neuronal networks. J Comp Neurol 514:240-258.

Bliss TV, Collingridge GL (1993) A synaptic model of memory: long-term potentiation in the hippocampus. Nature 361:31-39.

Bureau I, Shepherd GM, Svoboda K (2008) Circuit and plasticity defects in the developing somatosensory cortex of FMR1 knock-out mice. J Neurosci 28:5178-5188.

Chahrour M, Zoghbi HY (2007) The story of Rett syndrome: from clinic to neurobiology. Neuron 56:422-437.

Chang Q, Khare G, Dani V, Nelson S, Jaenisch R (2006) The disease progression of Mecp2 mutant mice is affected by the level of BDNF expression. Neuron 49:341-348.

Chao HT, Zoghbi HY, Rosenmund C (2007) MeCP2 controls excitatory synaptic strength by regulating glutamatergic synapse number. Neuron $56: 58-65$.

Chen HX, Otmakhov N, Lisman J (1999) Requirements for LTP induction by pairing in hippocampal CA1 pyramidal cells. J Neurophysiol 82:526-532.

Chen RZ, Akbarian S, Tudor M, Jaenisch R (2001) Deficiency of methylCpG binding protein-2 in CNS neurons results in a Rett-like phenotype in mice. Nat Genet 27:327-331.

Dani VS, Chang Q, Maffei A, Turrigiano GG, Jaenisch R, Nelson SB (2005) Reduced cortical activity due to a shift in the balance between excitation and inhibition in a mouse model of Rett syndrome. Proc Natl Acad Sci U S A 102:12560-12565.

Debanne D, Gähwiler BH, Thompson SM (1996) Cooperative interactions in the induction of long-term potentiation and depression of synaptic excitation between hippocampal CA3-CA1 cell pairs in vitro. Proc Natl Acad Sci U S A 93:11225-11230.

Douglas RJ, Martin KA (2004) Neuronal circuits of the neocortex. Annu Rev Neurosci 27:419-451.

Faber DS, Korn H (1991) Applicability of the coefficient of variation method for analyzing synaptic plasticity. Biophys J 60:1288-1294.

Feldman DE (2000) Timing-based LTP and LTD at vertical inputs to layer II/III pyramidal cells in rat barrel cortex. Neuron 27:45-56.

Frick A, Feldmeyer D, Sakmann B (2007) Postnatal development of synaptic transmission in local networks of L5A pyramidal neurons in rat somatosensory cortex. J Physiol 585:103-116.

Fukuda T, Itoh M, Ichikawa T, Washiyama K, Goto Y (2005) Delayed maturation of neuronal architecture and synaptogenesis in cerebral cortex of Mecp2-deficient mice. J Neuropathol Exp Neurol 64:537-544.

Giacometti E, Luikenhuis S, Beard C, Jaenisch R (2007) Partial rescue of $\mathrm{MeCP} 2$ deficiency by postnatal activation of MeCP2. Proc Natl Acad Sci U S A 104:1931-1936.

Guy J, Hendrich B, Holmes M, Martin JE, Bird A (2001) A mouse Mecp2null mutation causes neurological symptoms that mimic Rett syndrome. Nat Genet 27:322-326.

Guy J, Gan J, Selfridge J, Cobb S, Bird A (2007) Reversal of neurological defects in a mouse model of Rett syndrome. Science 315:1143-1147.

Hagberg B (1997) Condensed points for diagnostic criteria and stages in Rett syndrome. Eur Child Adolesc Psychiatry 6 [Suppl 1]:2-4.

Hanson JE, Blank M, Valenzuela RA, Garner CC, Madison DV (2007) The functional nature of synaptic circuitry is altered in area CA3 of the hippocampus in a mouse model of Down's syndrome. J Physiol 579:53-67.

Hattox AM, Nelson SB (2007) Layer V neurons in mouse cortex projecting to different targets have distinct physiological properties. J Neurophysiol 98:3330-3340.

Kirkwood A, Bear MF (1994) Hebbian synapses in visual cortex. J Neurosci 14:1634-1645.

Kishi N, Macklis JD (2004) MECP2 is progressively expressed in postmigratory neurons and is involved in neuronal maturation rather than cell fate decisions. Mol Cell Neurosci 27:306-321.

Kleschevnikov AM, Belichenko PV, Villar AJ, Epstein CJ, Malenka RC, Mobley WC (2004) Hippocampal long-term potentiation suppressed by increased inhibition in the Ts65Dn mouse, a genetic model of Down syndrome. J Neurosci 24:8153-8160.

Krieger P, Kuner T, Sakmann B (2007) Synaptic connections between layer $5 \mathrm{~B}$ pyramidal neurons in mouse somatosensory cortex are independent of apical dendrite bundling. J Neurosci 27:11473-11482.

Larkman A, Hannay T, Stratford K, Jack J (1992) Presynaptic release probability influences the locus of long-term potentiation. Nature 360:70-73.

Malinow R (1991) Transmission between pairs of hippocampal slice neurons: quantal levels, oscillations, and LTP. Science 252:722-724.

Malinow R, Tsien RW (1990) Presynaptic enhancement shown by wholecell recordings of long-term potentiation in hippocampal slices. Nature 346:177-180.

Markram H, Lübke J, Frotscher M, Sakmann B (1997a) Regulation of synaptic efficacy by coincidence of postsynaptic APs and EPSPs. Science 275:213-215.

Markram H, Lübke J, Frotscher M, Roth A, Sakmann B (1997b) Physiology and anatomy of synaptic connections between thick tufted pyramidal neurones in the developing rat neocortex. J Physiol 500:409-440.

Markram H, Rinaldi T, Markram K (2007) The intense world syndrome-an alternative hypothesis for autism. Front Neurosci 1:77-96.

Moretti P, Levenson JM, Battaglia F, Atkinson R, Teague R, Antalffy B, Armstrong D, Arancio O, Sweatt JD, Zoghbi HY (2006) Learning and memory and synaptic plasticity are impaired in a mouse model of Rett syndrome. J Neurosci 26:319-327.

Nelson ED, Kavalali ET, Monteggia LM (2006) MeCP2-dependent transcriptional repression regulates excitatory neurotransmission. Curr Biol 16:710-716.

Rinaldi T, Kulangara K, Antoniello K, Markram H (2007) Elevated NMDA receptor levels and enhanced postsynaptic long-term potentiation induced by prenatal exposure to valproic acid. Proc Natl Acad Sci U S A 104:13501-13506.

Rinaldi T, Silberberg G, Markram H (2008) Hyperconnectivity of local neocortical microcircuitry induced by prenatal exposure to valproic acid. Cereb Cortex 18:763-770. 
Sjöström PJ, Turrigiano GG, Nelson SB (2001) Rate, timing, and cooperativity jointly determine cortical synaptic plasticity. Neuron 32: 1149-1164.

Sjöström PJ, Turrigiano GG, Nelson SB (2003) Neocortical LTD via coincident activation of presynaptic NMDA and cannabinoid receptors. Neuron 39:641-654.

Sjöström PJ, Turrigiano GG, Nelson SB (2007) Multiple forms of long-term plasticity at unitary neocortical layer 5 synapses. Neuropharmacology 52:176-184.

Song S, Sjöström PJ, Reigl M, Nelson S, Chklovskii DB (2005) Highly nonrandom features of synaptic connectivity in local cortical circuits. PLoS Biol 3:e68.
Stearns NA, Schaevitz LR, Bowling H, Nag N, Berger UV, BergerSweeney J (2007) Behavioral and anatomical abnormalities in Mecp2 mutant mice: a model for Rett syndrome. Neuroscience 146:907-921.

Villard L (2007) MECP2 mutations in males. J Med Genet 44:417-423.

Zhou Z, Hong EJ, Cohen S, Zhao WN, Ho HY, Schmidt L, Chen WG, Lin Y, Savner E, Griffith EC, Hu L, Steen JA, Weitz CJ, Greenberg ME (2006) Brain-specific phosphorylation of $\mathrm{MeCP} 2$ regulates activity-dependent Bdnf transcription, dendritic growth, and spine maturation. Neuron 52:255-269.

Zoghbi HY (2003) Postnatal neurodevelopmental disorders: meeting at the synapse? Science 302:826-830. 